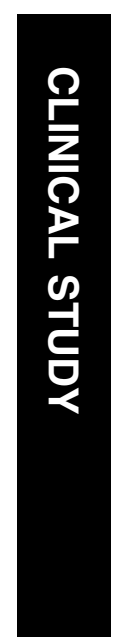

\title{
Retinal vascular occlusions occur more frequently in the more affected eye in exfoliation syndrome
}

${ }^{1}$ Department of Ophthalmology, Einhorn Clinical Research Center, New York Eye and Ear Infirmary, New York, NY, USA

${ }^{2}$ Retina Service, New York Eye and Ear Infirmary, New York, NY, USA

\section{${ }^{3}$ Department of} Ophthalmology, New York University School of Medicine, New York, NY, USA

${ }^{4}$ Department of Ophthalmology, New York Medical College, Valhalla, NY, USA

Correspondence: R Ritch, Glaucoma Service,

Department of

Ophthalmology,

The New York Eye and Ear Infirmary,

310 East 14th Street,

New York,

NY 10003

USA

Tel: + 11212673 5140;

Fax: + 112124208743

E-mail: ritchmd@

earthlink.net

Received: 27 February 2009 Accepted in revised form: 22 May 2009

Published online: 26 June 2009

\begin{abstract}
Purpose To evaluate the relationship between retinal vascular occlusions (RVOs) and exfoliation syndrome (XFS) in a cohort of patients with the two conditions.

Methods We reviewed the records of patients with XFS with or without glaucoma and any type of RVO between 1983 and 2007. Patients with prior incisional surgery or a history of uveitis were excluded. Data collected included demographics, systemic comorbidities, type of RVO, and intraocular pressure (IOP) before the RVO. Slit-lamp biomicroscopy regarding the presence of exfoliation material on the lens capsule and pupillary margin before the vascular event was used to evaluate the laterality and degree of XFS.

Results We identified 36 patients (mean age $78.4 \pm 8.3$ years, 19 women). Most patients were of European descent (34/36) and 20 (56\%) had no prior glaucoma diagnosis. The most common retinal vascular events were central retinal vein occlusion (18/36) and BRVO (10/36). Mean IOP between eyes with $(19.5 \pm 6.5 \mathrm{~mm} \mathrm{Hg})$ and without $(17.9 \pm 4.8 \mathrm{~mm} \mathrm{Hg}) \mathrm{RVO}$ was similar $(P=0.12)$. RVOs occurred more commonly in the eye with more pronounced XFS in $92 \%(33 / 36)$ of the cases. A similar agreement was found when considering patients with and without glaucoma separately $(94 \%$ (15/16) vs $90 \%$ $(18 / 20) ; P=0.83)$. In addition, no difference in the agreement percentage was observed when comparing patients with unilateral XFS $(87 \%(13 / 15))$ with all study patients $(P=0.87)$. Conclusions Retinal vascular occlusion in patients with XFS occurs most often in the affected or more severely affected eye.
\end{abstract}

TS Prata1 ${ }^{1}$, I Rozenbaum¹, CGV de Moraes ${ }^{1}$, VC Lima ${ }^{2}$, J Liebmann ${ }^{1,3}$ and R Ritch ${ }^{1,4}$
As vascular occlusions happened in patients with and without glaucoma in similar proportions, the presence of XFS seems to play an important role in these findings. Eye (2010) 24, 658-662; doi:10.1038/eye.2009.152; published online 26 June 2009

Keywords: XFS; RVOs; IOP; laterality

Introduction

Exfoliation syndrome (XFS) is the most common identifiable cause of open-angle glaucoma worldwide, accounting for the majority of cases in some countries. ${ }^{1,2}$ Although histopathological evaluation has shown that XFS is a bilateral disease, ${ }^{3-6}$ the eyes are often asymmetrically involved. ${ }^{7-12}$ Exfoliation material (XFM) is found not only in ocular tissues, ${ }^{13-15}$ and several systemic disorders have been associated with XFS, such as peripheral vascular occlusions, increased carotid stiffness, coronary artery disease, stroke, Alzheimer's disease, and hearing loss. ${ }^{16-23}$

The pathogeneses and clinical features of acute retinal vascular occlusions (RVOs) are still not well understood. ${ }^{24,25}$ Central retinal vein occlusion (CRVO) has been described in eyes with XFS, even when exfoliative glaucoma (XFG, a known risk factor) was not present. ${ }^{26,27}$ As ocular involvement by XFS and XFG is often clinically unilateral or asymmetric, ${ }^{7-12}$ the severity of ocular findings and its laterality could be associated with the side of occurrence of vascular occlusions. To test this hypothesis, we investigated the relationship between XFS/XFG and RVO in a cohort of patients with the two conditions. 


\section{Patients and methods}

This retrospective study adhered to the tenets of the Declaration of Helsinki and the New York Eye and Ear Infirmary Institutional Review Board guidelines.

\section{Patients}

We reviewed all charts of patients presenting with XFS with or without ocular hypertension or glaucoma associated with RVO between 1983 and 2007. Only patients with clinically evident XFS on slit-lamp examination, and not any patients diagnosed to have XFS on the basis of conjunctival biopsy, were included. After excluding patients with incisional intraocular surgery or any other eye disease before the RVO, we enrolled 45 patients. All patients with XFG were under topical antiglaucoma treatment.

Data collected included age, gender, race, self-reported systemic comorbidities, and the type of RVO. Information from slit-lamp biomicroscopy regarding the presence of XFM on the pupillary margin and lens capsule (with pupil dilation), ${ }^{28,29}$ and intraocular pressure (IOP) was recorded (last IOP reading before the RVO onset).

A secondary analysis was carried out to determine and select which eye was affected or more severely affected by XFS. Patients had to meet one of the following laterality/asymmetry criteria: (i) clinically unilateral XFS; (ii) marked asymmetry in bilateral XFS (differences in the amount of XFM between eyes are graded in the charts using + marks); (iii) unilateral XFG (only one eye presenting glaucomatous changes in visual field and/or optic disc photos); (iv) marked asymmetry in bilateral XFG cases (the eye with more advanced glaucomatous damage based on visual field and/or optic disc photos). Standard automated perimetry (Humphrey, Carl Zeiss Meditec, Inc., Dublin, CA, USA) results and optic disc photographs were assessed to determine the eye with more advanced XFG. Asymmetric XFG was defined as a difference in cup-to-disc ratio $>0.2$ and/or a difference in the mean deviation of $3.0 \mathrm{~dB}$.

\section{Statistical analysis}

Comparison of the IOP (based on one single baseline IOP measurement) between fellow eyes was performed using Student's paired $t$-test assuming statistical significance for $P<0.05$. The agreement between the eye involved in the RVO and the affected or more affected eye by XFS/XFG was then calculated for all patients. The same analysis was done comparing patients with and without glaucoma separately, and including patients with unilateral XFS only $\left(\chi^{2}\right.$-test).

\section{Results}

Thirty-six patients fulfilled the asymmetry criteria. Baseline characteristics are presented in Table 1. Mean age at the time of the occlusion was $78.4 \pm 8.3$ years. Most patients were of European descent (34/36), 19 (53\%) were women and $20(56 \%)$ had no prior glaucoma history. In 15 patients (42\%), XFS was clinically unilateral at the time of presentation. Five patients $(14 \%)$ had bilateral asymmetric XFS, eight (22\%) had unilateral XFG, and eight $(22 \%)$ had bilateral asymmetric XFG. The most common vascular occlusion was CRVO (18/36), and $14 \%$ of the patients had an arterial occlusion (5/36). During the follow-up of these 36 patients, neovascular glaucoma subsequently developed in seven cases (six after CRVO). One patient, who had a CRVO in the eye with more advanced XFG, developed a CRVO in the fellow eye 2 years later. A second patient, who had a central retinal artery occlusion in the only eye with XFS, developed a non-arteritic anterior ischaemic optic neuropathy (NAION) in the fellow eye 6 years later. Interestingly, XFM deposits were noted in this eye 2 years before the NAION onset.

In $92 \%$ of the cases $(33 / 36)$, the eye in which the RVO occurred was also the affected or more severely affected XFS eye. Comparing the mean IOP (average of baseline measurement of all patients) between involved (with RVO) and uninvolved (no RVO) eyes, we found no significant difference $(19.5 \pm 6.5$ vs $17.9 \pm 4.8 \mathrm{~mm} \mathrm{Hg}$, $P=0.12$ ). In addition, a similar agreement was found when considering patients with and without glaucoma separately (94\% (15/16) vs 90\% $(18 / 20) ; P=0.83))$.

When considering patients with unilateral XFS only, a strong agreement (87\% (13 out of 15 cases)) was observed between the eye with XFS and the eye in which the RVO occurred. In addition, no difference in the percentage of agreement was observed when comparing these cases (unilateral XFS) with all study patients (87 vs 92\%; $P=0.87)$. Finally, in the five eyes with retinal arterial occlusion, the eye in which the RVO occurred was always the affected or more severely affected XFS eye.

\section{Discussion}

We found that RVO tends to occur in the affected or more severely affected eye in patients with XFS or XFG. This significant agreement was also observed when considering patients with and without glaucoma separately, and when analyzing patients with unilateral XFS only. This is the first report to investigate the relationship between the laterality and severity of XFS/ XFG with the development of different types of RVO.

An association of XFS with RVO has been previously described. Saatci et $a l^{27}$ clinically documented XFS 
Table 1 Characteristics of patients with XFS and RVO

\begin{tabular}{lc}
\hline Variables & Study patients $(\mathrm{n}=36)$ \\
\hline Gender (male/female) & $19 / 17$ \\
Race (ED/H) & $34 / 2$ \\
Mean age \pm SD (years) & $78.4 \pm 8.3$ \\
Glaucoma before RVO & 16 \\
Developed neovascular glaucoma & 7 \\
Retinal vascular occlusion types & \\
Central retinal vein occlusion & 18 \\
Branch retinal vein occlusion & 10 \\
Hemiretinal vein occlusion & 3 \\
Central retinal artery occlusion & 4 \\
Branch retinal artery occlusion & 1 \\
Systemic comorbities & \\
Systemic hypertension & 23 \\
Diabetes & 8 \\
\hline
\end{tabular}

ED, European descent; H, Hispanic; RVO, retinal vascular occlusion; XFS, exfoliation syndrome.

findings in $8.2 \%$ of eyes with BRVO and $20.8 \%$ with CRVO, as opposed to $5.2 \%$ in control eyes. In another retrospective study, XFS was found in $6.0 \%$ of BRVO eyes and $6.9 \%$ of CRVO eyes. ${ }^{26}$ XFS may be present but not yet visible by slit-lamp examination. ${ }^{28,30-32}$ We have recently investigated the presence of XFS in 36 CRVO eyes using electron microscopy, and a clinical diagnosis or a positive conjunctival biopsy result was found in 22 eyes. ${ }^{33} \mathrm{We}$ hypothesised that the connection between XFS and RVO might depend on the extent of involvement of an eye by XFS. The findings of this study corroborate this hypothesis.

The reason why an RVO occurs most often in the affected or more severely affected eye in patients with XFS could possibly be attributed to a combination of factors. Structural vascular abnormalities documented in eyes with XFS could be involved. ${ }^{34-36}$ Immunohistochemistry analysis showed marked perivascular deposits of XFM involving the basement membrane of iris blood vessels in the involved eyes, but not in the uninvolved fellow eyes. ${ }^{37}$ In addition, ocular blood flow ${ }^{38}$ and ipsilateral carotid blood flow ${ }^{39}$ have been reported to be reduced in the involved eye. Abnormal homocysteine metabolism could be an important factor as well. Hyperhomocysteinaemia, which is a major risk factor for vascular diseases (including venous thrombosis), was reported to be more common in XFS and XFG patients than in healthy controls in several studies. ${ }^{39-42}$ Finally, endothelin-1, which is a potent vasoconstrictor, seems to be significantly increased in the aqueous of XFS eyes, which could contribute to the obliterative vasculopathy. ${ }^{43}$
Elevated IOP and glaucoma have also been suggested as a cause for the association of XFS with CRVO. ${ }^{24,26,44}$ Gillies et $a l^{44}$ also found a strong agreement between the involved eye and the CRVO site in 10 patients with unilateral XFS, and attributed their findings to the higher IOP observed in the affected eyes. In our larger study, over $50 \%$ of the patients did not carry a diagnosis of glaucoma or ocular hypertension before developing RVO. In addition, average IOP for involved and uninvolved eyes was similar, and we found no difference in the agreement between eyes with and without glaucoma. These facts suggest that XFS itself may play an important role in the vascular occlusion process.

It is important to stress some specific characteristics of this study. First, all the XFG patients in the study were on antiglaucoma treatment, which could have masked a possible difference in IOP between affected and unaffected eyes. Second, the retrospective nature of our study may have limited a reliable assessment of the XFS asymmetry. However, performing this study prospectively would not be feasible. To try to overcome this limitation, we did a separate analysis including patients with unilateral XFS only. No significant difference in the percentage of agreement was observed when comparing these cases to all study patients. Third, as arterial occlusions comprised $<15 \%$ of our cases, findings related to this type of vascular event have limited clinical significance. Finally, it is well known that XFG patients tend to present a higher diurnal IOP fluctuation than primary open-angle glaucoma patients. ${ }^{45}$ As we used only one measurement to determine baseline IOP, we cannot exclude a possible IOP spike. Despite no statistically significant difference in mean baseline IOP (average IOP considering all eyes) between groups $(1.6 \mathrm{~mm} \mathrm{Hg})$, this difference could be clinically important in increasing the risk of vascular occlusions in these eyes.

In summary, our data suggest that in patients with XFS that present an RVO, the latter occurs in the affected or more severely affected eye by XFS/XFG in the majority of the cases. As this happens in patients with and without glaucoma in similar proportions, the presence of XFS seems to play an important role in these findings. Histopathological studies searching for ultrastructural vascular abnormalities in XFS patients with and without $\mathrm{RVO}$ are required to better explain this association.

\section{Acknowledgements}

Supported in part by a Horizon award from Allergan, Inc. Irvine, CA, USA and the Joseph Cohen Research Fund of the New York Glaucoma Research Institute, New York, NY, USA. 


\section{References}

1 Ritch R. Exfoliation syndrome: the most common identifiable cause of open-angle glaucoma. J Glaucoma 1994; 3: 176-178.

2 Forsius H. Exfoliation syndrome in various ethnic populations. Acta Ophthalmol Suppl 1988; 184: 71-85.

3 Speakman JS, Ghosh M. The conjunctiva in senile lens exfoliation. Arch Ophthalmol 1976; 94: 1757-1759.

4 Madden JG, Crowley MJ. Factors in the exfoliation syndrome. Br J Ophthalmol 1982; 66: 432-437.

5 Hirvelä H, Luukinen H, Laatikainen L. Prevalence and risk factors of lens opacities in the elderly in Finland. A population-based study. Ophthalmology 1995; 102: 108-117.

6 Hammer T, Schlötzer-Schrehardt U, Naumann GO. Unilateral or asymmetric pseudoexfoliation syndrome? An ultrastructural study. Arch Ophthalmol 2001; 119: 1023-1031.

7 Brooks AM, Gillies WE. The presentation and prognosis of glaucoma in pseudoexfoliation of the lens capsule. Ophthalmology 1988; 95: 271-276.

8 Vesti E, Kivelä T. Exfoliation syndrome and exfoliation glaucoma. Prog Retin Eye Res 2000; 19: 345-368.

9 Crittendon JJ, Shields MB. Exfoliation syndrome in the southeastern United States. II. Characteristics of patient population and clinical course. Acta Ophthalmol Suppl 1988; 184: 103-106.

10 Pohjanpelto PE. The fellow eye in unilateral hypertensive pseudoexfoliation. Am J Ophthalmol 1973; 75: 216-220.

11 Puska P, Raitta C. Exfoliation syndrome as a risk factor for optic disc changes in nonglaucomatous eyes. Graefes Arch Clin Exp Ophthalmol 1992; 230: 501-504.

12 Tomita G, Puska P, Raitta C. Interocular differences in optic disc configuration in the unilateral exfoliation syndrome. Acta Ophthalmol (Copenh) 1994; 72: 162-167.

13 Schlötzer-Schrehardt UM, Koca MR, Naumann GO, Volkholz H. Pseudoexfoliation syndrome. Ocular manifestation of a systemic disorder? Arch Ophthalmol 1992; 110: 1752-1756.

14 Ritch R, Schlötzer-Schrehardt U. Exfoliation syndrome. Surv Ophthalmol 2001; 45: 265-315.

15 Streeten BW, Li ZY, Wallace RN, Eagle Jr RC, Keshgegian AA. Pseudoexfoliative fibrillopathy in visceral organs of a patient with pseudoexfoliation syndrome. Arch Ophthalmol 1992; 110: 1757-1762.

16 Mitchell P, Wang JJ, Smith W. Association of pseudoexfoliation syndrome with increased vascular risk. Am J Ophthalmol 1997; 124: 685-687.

17 Linnér E, Popovic V, Gottfries CG, Jonsson M, Sjögren M, Wallin A. The exfoliation syndrome in cognitive impairment of cerebrovascular or Alzheimer's type. Acta Ophthalmol Scand 2001; 79: 283-285.

18 Atalar PT, Atalar E, Kilic H, Abbasoglu OE, Ozer N, Aksöyek $\mathrm{S}$ et al. Impaired systemic endothelial function in patients with pseudoexfoliation syndrome. Int Heart J 2006; 47: 77-84.

19 Visontai Z, Merisch B, Kollai M, Holló G. Increase of carotid artery stiffness and decrease of baroreflex sensitivity in exfoliation syndrome and glaucoma. Br J Ophthalmol 2006; 90: 563-567.

20 Yazdani S, Tousi A, Pakravan M, Faghihi AR. Sensorineural hearing loss in pseudoexfoliation syndrome. Ophthalmology 2008; 115: 425-429.

21 Bojić L, Ermacora R, Polić S, Ivanisević M, Mandić Z, Rogosić V et al. Pseudoexfoliation syndrome and asymptomatic myocardial dysfunction. Graefes Arch Clin Exp Ophthalmol 2005; 243: 446-449.

22 Andrikopoulos GK, Mela EK, Georgakopoulos CD, Papadopoulos GE, Damelou AN, Alexopoulos DK et al. Pseudoexfoliation syndrome prevalence in Greek patients with cataract and its association to glaucoma and coronary artery disease. Eye 2009; 23: 442-447.

23 Irkec M. Exfoliation and carotid stiffness. Br J Ophthalmol 2006; 90: 529-530.

24 David R, Zangwill L, Badarna M, Yassur Y. Epidemiology of retinal vein occlusion and its association with glaucoma and increased intraocular pressure. Ophthalmologica 1988; 197: 69-74.

25 Hayreh SS. Prevalent misconceptions about acute retinal vascular occlusive disorders. Prog Retin Eye Res 2005; 24: 493-519.

26 Cursiefen C, Händel A, Schönherr U, Naumann GO. Pseudoexfoliation syndrome in patients with retinal vein branch and central vein thrombosis. Klin Monatsbl Augenheilkd 1997; 211: 17-21.

27 Saatci OA, Ferliel ST, Ferliel M, Kaynak S, Ergin MH. Pseudoexfoliation and glaucoma in eyes with retinal vein occlusion. Int Ophthalmol 1999; 23: 75-78.

28 Prince AM, Streeten BW, Ritch R, Dark AJ, Sperling M. Preclinical diagnosis of pseudoexfoliation syndrome. Arch Ophthalmol 1987; 105: 1076-1082.

29 Prince AM, Ritch R. Clinical signs of the pseudoexfoliation syndrome. Ophthalmology 1986; 93: 803-807.

30 Krause U, Tarkkanen A. Cataract and pseudoexfoliation. A clinicopathological study. Acta Ophthalmol (Copenh) 1978; 56: 329-334.

31 Konstas AG, Jay JL, Marshall GE, Lee WR. Prevalence, diagnostic features, and response to trabeculectomy in exfoliation glaucoma. Ophthalmology 1993; 100: 619-627.

32 Ritch R. Exfoliation syndrome: clinical findings and occurrence in patients with occludable angles. Trans Am Ophthalmol Soc 1994; 92: 845-944.

33 Ritch R, Prata TS, De Moraes CGV, Vessani RM, Costa VP, Konstas AGP et al. Association of exfoliation syndrome and central retinal vein occlusion: an ultrastructural analysis. Acta Ophthalmol Scand 2009 (in press).

34 Eagle Jr RC, Font RL, Fine BS. The basement membrane exfoliation syndrome. Arch Ophthalmol 1979; 97: 510-515.

35 Küchle M, Schlötzer-Schrehardt U, Naumann GO. Occurrence of pseudoexfoliative material in parabulbar structures in pseudoexfoliation syndrome. Acta Ophthalmol (Copenh) 1991; 69: 124-130.

36 Schlötzer-Schrehardt U, Küchle M, Naumann GO. Electronmicroscopic identification of pseudoexfoliation material in extrabulbar tissue. Arch Ophthalmol 1991; 109: 565-570.

37 Kivelä T, Hietanen J, Uusitalo M. Autopsy analysis of clinically unilateral exfoliation syndrome. Invest Ophthalmol Vis Sci 1997; 38: 2008-2015.

38 Sibour G, Finazzo C, Boles Carenini A. Monolateral pseudoexfoliatio capsulae: a study of choroidal blood flow. Acta Ophthalmol Scand Suppl 1997; 224: 13-14.

39 Maral H, Yüksel N, aba ${ }^{\circ}$ VL, Dillioğlugil MO, Cağlar Y. Homocysteine and nitric oxide levels in plasma of patients with pseudoexfoliation syndrome, pseudoexfoliation glaucoma, and primary open-angle glaucoma. Graefes Arch Clin Exp Ophthalmol 2005; 243: 677-683.

40 Saricaoglu MS, Karakurt A, Sengun A, Hasiripi H. Plasma homocysteine levels and vitamin B status in patients with Pseudoexfoliation syndrome. Saudi Med J 2006; 27: 833-837. 
41 Leibovitch I, Kurtz S, Shemesh G, Goldstein M, Sela BA, Lazar $\mathrm{M}$ et al. Hyperhomocystinemia in pseudoexfoliation glaucoma. J Glaucoma 2003; 12: 36-39.

42 Vessani RM, Ritch R, Liebmann JM, Jofe M. Plasma homocysteine is elevated in patients with exfoliation syndrome. Am J Ophthalmol 2003; 136: 41-46.

43 Koliakos GG, Konstas AG, Schlötzer-Schrehardt U, Hollo G, Mitova D, Kovatchev D et al. Endothelin-1 concentration is increased in the aqueous humour of patients with exfoliation syndrome. Br J Ophthalmol 2004; 88: 523-527.

44 Gillies WE, Brooks AM. Central retinal vein occlusion in pseudoexfoliation of the lens capsule. Clin Experiment Ophthalmol 2002; 30: 176-187.

45 Konstas AG, Mantziris DA, Stewart WC. Diurnal intraocular pressure in untreated exfoliation and primary open-angle glaucoma. Arch Ophthalmol 1997; 115: 182-185. 